\title{
Upregulated solute carrier family 37 member 1 in colorectal cancer is associated with poor patient outcome and metastasis
}

\author{
DAIKI KIKUCHI, MOTONOBU SAITO, KATSUHARU SAITO, YOHEI WATANABE, \\ YOSHIKO MATSUMOTO, YASUYUKI KANKE, HISASHI ONOZAWA, SUGURU HAYASE, WATARU SAKAMOTO, \\ TERUHIDE ISHIGAME, TOMOYUKI MOMMA, SHINJI OHKI and SEIICHI TAKENOSHITA \\ Department of Organ Regulatory Surgery, Fukushima Medical University School of Medicine, \\ Fukushima 960-1295, Japan
}

Received July 27, 2016; Accepted March 3, 2017

DOI: $10.3892 / \mathrm{ol} .2017 .7559$

\begin{abstract}
Solute carrier (SLC) drug transporters exchange various molecules without energy from adenosine triphosphate hydrolysis, indicating an association with anticancer drug resistance. However, the expression and role of SLC transporters in malignant tumors has not yet been fully elucidated. Therefore, in the current study, the expression of SLC37A family genes was evaluated in patients with colorectal cancer (CRC), and it was revealed that SLC family 37 member 1 (SLC37A1) expression was significantly increased in tumorous tissues compared with that in non-tumorous tissues. The cases with upregulated expression of SLC37A1 by immunohistochemical staining were significantly associated with positive venous invasion and liver metastasis. Furthermore, upregulated SLC37A1 expression was associated with poor overall survival time in the present cohort. These results indicated that SLC37A1 is involved in the hematogenous metastasis of CRC. To investigate whether SLC37A1 is associated with hematogenous metastasis and glycolipid metabolism, SLC37A1 was knocked down in colon cancer cells, and the expression of sialyl Lewis A and sialyl Lewis X was observed to be decreased. In summary, upregulation of SLC37A1 was observed in patients with $\mathrm{CRC}$, and was associated with poor patient outcomes and survival. To the best of our knowledge, the present study is the first to propose a key role of SLC37A1 in CRC, and additional studies are warranted to reveal the functional role of SLC37A1 in CRC development.
\end{abstract}

Correspondence to: Dr Seiichi Takenoshita, Department of Organ Regulatory Surgery, Fukushima Medical University School of Medicine, 1 Hikarigaoka, Fukushima 960-1295, Japan

E-mail: takenoss@fmu.ac.jp

Key words: colorectal cancer, solute carrier family 37 member 1, sialyl Lewis A, sialyl Lewis X, venous invasion, liver metastasis

\section{Introduction}

Colorectal cancer (CRC) is one of the leading causes of cancer-associated mortality in the USA and East Asia (1-3). While segmental surgical resection of the colon or rectum is the standard treatment, surgery alone is not sufficient to cure the majority of patients with CRC (4). Patients who experience local or distant recurrences following surgery require chemotherapy (4). Furthermore, patients with stage III CRC, as well as selected patients with stage II, also require adjuvant chemotherapy to reduce the risk of recurrence (5). 5-Fluorouracil (5-FU) has been used as a standard chemotherapy regimen in the past few decades and remains a key drug in several recently introduced combination chemotherapies (6). FOLFOX [folinic acid (LV), 5-FU and oxaliplatin] and FORFIRI (LV, 5-FU and irinotecan) provide a high response rate, and their therapeutic effect is evident in numerous patients with CRC (7-10). However, drug resistance to these combination chemotherapies has finally occurred and may be a major problem in the treatment of CRC (11).

One of the mechanisms of drug resistance is the transportation of anticancer drugs out of cells, and the major drug transporters are the adenosine triphosphate (ATP)-binding cassette (ABC) and solute carrier (SLC) superfamilies (12). $\mathrm{ABC}$ transporters contain membrane proteins that translocate a variety of substrates across extra- and intracellular membranes, and use the energy from ATP binding/hydrolysis to transport multiple types of drugs, including anticancer agents (13). In vitro studies have revealed that more than half of $\mathrm{ABC}$ transporters are associated with chemotherapy failure (14). The most characterized $A B C$ transporter is ABCB1 (also known as multidrug resistance protein 1), the overexpression of which in tumor cells has been associated with drug resistance to molecularly targeted and chemotherapy drugs $(15,16)$. By contrast, SLC drug transporters do not rely on ATP and uptake small molecules into cells (12). While cancer cells express several different types of $\mathrm{ABC}$ transporters to gain drug resistance (17), the expression of SLC transporters in malignant tumors remains unknown. SLC transporters include almost 400 members organized into 52 families, and it has been reported that certain transporters, including SLC22A6 and SLC22A8 from the SLC22 family, 
transport methotrexate (18). The present study suggested that SLC transporters may also be associated with drug resistance (18). However, the molecular functions of the majority of SLC transporters in cancer remain to be elucidated.

The SLC37 family consists of four proteins, namely A1, A2, A3 and A4, which are involved in sugar-phosphate exchange (19). SLC37A4 is known as a glucose-6-phosphate (G6P) transporter that performs an important role in glucose production (19); however, the functional role of other SLC37 family genes is not yet fully understood. However, since this transporter functions in glycolipid metabolism, it has been hypothesized that abundant expression of SLC37 family genes is involved in cancer development (19). It is known that oncogenes and tumor-suppressor genes dysregulate glucose transport and energy metabolism pathways due to satisfy the requirement of a large amount of glucose for cancer cells $(20,21)$. Therefore, a previous study investigated the roles of SLC transporters and expected them to be a useful therapeutic target (12).

In the current study, SLC37 family gene expression was investigated in patients with CRC. Furthermore, to clarify the functional role of SLC37 genes, the association between SLC37 gene expression and glycolipid metabolism was investigated.

\section{Materials and methods}

Clinical samples of patients. A total of 231 surgical specimens obtained from 231 patients with CRC who had undergone surgical resection at Fukushima Medical University Hospital (Fukushima, Japan) between January 1991 and December 2007 were included in the present study. In 10 cases, messenger RNAs (mRNAs) were extracted from cancer tissues and adjacent non-tumor tissues. Information regarding age, sex, tumor-node-metastasis stage and pathological diagnosis, including lymphatic and venous invasion, was retrospectively collected (Tables III and IV). The carcinomas at the time of primary tumor resection were staged according to the Union for International Cancer Control classification (7th classification) $(22,23)$. Written informed consent was obtained from all patients. The present study was approved by the ethics committee of Fukushima Medical University (approval no. 1476).

Cell line culture. The colon cancer cell lines (SW620, LoVo, SW480, HCT116, SW48, HCT15, RKO, SW837, COLO-201, COLO-205, LS174T and LS180) used in the present study were originally obtained from the American Type Culture Collection (Manassas, VA, USA). The cells were cultured in the recommended media (Dulbecco's modified Eagle's medium (Thermo Fisher Scientific, Inc., Waltham, MA, USA) for RKO, LS174T and LS180 and RPMI-1640 medium (Sigma-Aldrich; Merck KGaA, Darmstadt, Germany) for SW480, Lovo, HCT15, SW48, SW620 and HCT116 supplemented with 10\% fetal bovine serum (Thermo Fisher Scientific, Inc.). The monolayer cells were maintained in a $37^{\circ} \mathrm{C}$ incubator with $5 \% \mathrm{CO}_{2}$, observed regularly under a light microscope (magnification, x40) and subcultured when they reached 80-90\% confluency.

Reverse transcription-quantitative polymerase chain reaction $(R T-q P C R)$. Total RNA was extracted from the tissues and cells using TRIzol reagent (Thermo Fisher Scientific, Inc., Waltham, MA, USA) according to the manufacturer's protocol, as previously described (24). Complementary DNA (cDNA) was synthesized from $5 \mu \mathrm{g}$ of total RNA with a random hexamer using the SuperScript III First-Strand Synthesis System (Thermo Fisher Scientific, Inc.). The cDNAs from the CRC tissues were used for the measurement of four SLC37 family genes. $\beta$-actin was used as an internal control. Primer sequences are shown in Table I. To quantify the gene expression, the band was scanned, and a densitometry analysis was performed using the software ImageJ version $1.51 \mathrm{k}$ (National Institutes of Health, Bethesda, MD, USA). The experiment was repeated at least two times.

RT-qPCR amplification of the SLC37A1 gene was performed with the GeneAmp PCR System 9700 (Thermo Fisher Scientific, Inc.) at $94^{\circ} \mathrm{C}$ initial denaturation for $5 \mathrm{~min}$, followed by $25-35$ cycles of $94^{\circ} \mathrm{C}$ for $1 \mathrm{~min}, 55-60^{\circ} \mathrm{C}$ for $45 \mathrm{sec}$ and $72^{\circ} \mathrm{C}$ for $45 \mathrm{sec}$. These cDNAs were used for the measurement of gene expression by real-time PCR with an Applied Biosystems ${ }^{\circledR} 7500$ Real-time PCR system (Thermo Fisher Scientific, Inc.) using TaqMan ${ }^{\circledR}$ probes that were purchased from Thermo Fisher Scientific, Inc., and $\beta$-actin was used as an internal control: SLC37A1 (catalog no., Hs00375251_m1) and $\beta$-actin (catalog no., Hs99999903_m1). Relative SLC37A1 gene expression was calculated using the $2^{-\Delta \Delta \mathrm{Cq}}$ method (25), according to the supplier's protocol (Thermo Fisher Scientific, Inc.). This sets the average control expression at 1 . This experiment was repeated this three times.

Immunohistochemical (IHC) staining and evaluation. IHC staining was carried out on paraffin-embedded histological sections (4- $\mu \mathrm{m}$ thick) using a polymer peroxidase method in which colon cancer specimens were fixed in $20 \%$ phosphate-buffered formalin $(\mathrm{pH} 7.4)$ at room temperature overnight. Following deparaffinization with xylene and rehydration using alcohol-water mixtures, prior to heat treatment in $10 \mathrm{mM}$ citric acid ( $\mathrm{pH}$ 6.0) for antigen retrieval, the sections were treated with $0.3 \%$ hydrogen peroxide in methanol for $30 \mathrm{~min}$ at room temperature to block endogenous peroxidase activity. Subsequent to rinsing in PBS, the sections were incubated with anti-SLC37A1 antibody (catalog no., HPA030418; dilution, 1:200; Atlas Antibodies, Bromma, Sweden) at $4^{\circ} \mathrm{C}$ overnight. An additional wash in PBS was followed by treatment with a peroxidase-labeled polymer conjugated to goat anti-rabbit immunoglobulins (Dako EnVision+ System-HRP Labelled Polymer; ready-to-use; catalog no., K4003; Dako; Agilent Technologies, Inc., Santa Clara, CA, USA), as the secondary antibody for $30 \mathrm{~min}$ at room temperature. The staining was visualized with 3,3'-diaminobenzidine, followed by counterstaining with hematoxylin.

Expression of SLC37A1 proteins was evaluated using optical microscopy (BX43; Olympus Corporation, Tokyo, Japan) as positive when detected in the nucleus of the tumorous tissue, but not in the nucleoli, cytoplasm or endoplasmic reticulum, and the total field of view was observed at x400 magnification. Blinded to the origin of the features and clinical outcomes, the staining of each specimen was evaluated by two independent researches (D.K. and K.S.). Rates were classified as follows: $0-5 \%, 0 ; 6-20 \%, 1 ; 21-50 \%, 2$; and $51-100 \%, 3$. Staining intensity was scored as 0 (negative), 1 (weak), 2 (moderate) and 3 
Table I. Primers used for reverse transcription-quantitative polymerase chain reaction analysis.

\begin{tabular}{llll}
\hline Gene & \multicolumn{1}{c}{ Forward primer (5'-3') } & \multicolumn{1}{c}{ Reverse primer (5'-3') } \\
\hline SLC37A1 & TCATTGATCGCTGGCTACTG & CAAGGTGACCACATTCGTG & 388 \\
SLC37A2 & CCTTTCACCTCGCTCTTTG & ATTTGGCCACAGTCTCAAGG & 446 \\
SLC37A3 & CTCGGGTATTGAGGCAGAAG & AGCCTGTCTCCTTAGCACGA & 445 \\
SLC37A4 & TCAATCGCAAGACCTTCTCC & CATCAGCAGGTTCATTGTGG \\
$\beta$-actin & GCTCGTCGTCGACAACGGCTC & CAAACATGATCTGGGTCATCTTCTC & 395 \\
\hline
\end{tabular}

SLC, solute carrier.

Table II. Tumor/non-tumor ratio ${ }^{\mathrm{a}}$ of SLC37A gene expression.

\begin{tabular}{lccccccccccc}
\hline & \multicolumn{10}{c}{ Case } \\
\cline { 2 - 13 } Gene & 1 & 2 & 3 & 4 & 5 & 6 & 7 & 8 & 9 & 10 & Mean \\
\hline SLC37A1 & 1.35 & 1.43 & 1.27 & 0.42 & 0.84 & 0.84 & 2.23 & 3.98 & 1.70 & 0.67 & 1.48 \\
SLC37A2 & 0.91 & 1.79 & 1.01 & 0.74 & 1.24 & 1.54 & 1.28 & 0.65 & 0.86 & 1.08 & 1.11 \\
SLC37A3 & 1.74 & 1.11 & 1.29 & 1.32 & 0.87 & 0.89 & 0.69 & 0.93 & 1.10 & 1.06 & 1.10 \\
SLC37A4 & 0.95 & 1.56 & 0.95 & 1.45 & 0.67 & 0.89 & 1.92 & 1.01 & 1.24 & 1.22 & 1.19 \\
\hline
\end{tabular}

${ }^{a}$ The tumor/non-tumor ratio was calculated from the quantified data of tumor (tumor/ $\beta$-actin) and non-tumor (non-tumor/ $\beta$-actin) expression .

(strong). Positive staining was defined by a score of $>2$, while negative staining was a score of 0 or 1 .

Small interfering RNA (siRNA) transfection. A knockdown experiment was performed using the siRNA method with SLC37A1 Stealth RNAi oligos HSS1227622 (SLC37A1-siRNA1) and HSS182427 (SLC37A1-siRNA2) (Thermo Fisher Scientific, Inc.), and Lipofectamine RNAiMAX (Thermo Fisher Scientific, Inc.) according to the manufacturer's protocol. Stealth RNAi siRNA negative control (NC-siRNA) (catalog no. 12935-300; Thermo Fisher Scientific, Inc.) was used as control. One day prior to transfection, a CRC cell line, LS180, was seeded at $5 \times 10^{5}$ cells per well on a $6-\mathrm{cm}$ plate. Transfection with a final concentration of $40 \mathrm{nM}$ siRNA was conducted when the cell density was $30-50 \%$ on the plates, and the plates were then incubated for $48 \mathrm{~h}$ at $37^{\circ} \mathrm{C}$.

Fluorescence-activated cellsorting (FACS).FACS was employed to characterize sialyl Lewis A and sialyl Lewis X protein expression levels. The anti-sialyl Lewis A antibody (catalog no., 2D3; Seikagaku Kogyo Co., Ltd., Tokyo, Japan) and anti-sialyl Lewis X (catalog no., 2H5; Seikagaku Kogyo Co., Ltd.) were prepared as previously described (26). For flow-cytometric analysis, the cells were harvested at a semi-confluent stage and stained with a fluorescein isothiocyanate-conjugated secondary antibody for $30 \mathrm{~min}$ at $25^{\circ} \mathrm{C}$ (diliution, 1:250; Chemicon; EMD Millipore, Billerica, MA, USA) prior to analysis using FACSCanto II (BD Biosciences, San Jose, CA, USA).

Statistical analysis. Statistical analysis was carried out with an unpaired Student's t-test using GraphPad Prism v5.0 (GraphPad Software, Inc., La Jolla, CA, USA). Survival curves were generated by the Kaplan-Meier method and compared by log-rank tests. Univariate and multivariate Cox regression analyses were performed by JMP 10 software (SAS Institute, Inc., Cary, NC, USA). $\mathrm{P}<0.05$ was considered to indicate a statistically significant difference.

\section{Results}

Upregulated SLC37A1 expression is associated with worse patient outcomes. To investigate SLC37A family gene expression in CRC, the mRNA expression of each cluster gene was investigated in 10 representative cases using RT-qPCR (Fig. 1A and Table I). While all SLC37A family genes were detected in tumor and adjacent non-cancerous tissue, SLC37A1 expression appeared to be markedly increased in tumor tissue compared with its expression in non-tumor tissue (Table II). To investigate these comparisons, SLC37A1 protein expression was evaluated by IHC staining in 231 patients with CRC (Fig. 1B). SLC37A1 expression was positive in 157 cases $(68.0 \%)$ and negative in 74 cases $(32.0 \%)$. SLC37A1 expression was observed in the nucleus of cancer cells of patients with CRC.

SLC37A1 expression level was then compared with clinicopathological factors in patients with CRC (Table III). The SLC37A1 expression level was not associated with age, sex, stage, tumor location, histology, depth of invasion, lymphatic invasion or lymph node metastasis. However, positive expression of SLC37A1 was significantly associated with positive venous invasion $(\mathrm{P}=0.034)$ and liver metastasis $(\mathrm{P}=0.013)$. Based on the Kaplan-Meier analysis, positive expression of SLC37A1 was significantly associated with worse overall survival ( $\mathrm{P}=0.019)$ (Fig. 1C). This association was marginally significant $(\mathrm{P}=0.085)$ and independent of all clinical covariates 
Table III. Clinicopathological factors and SLC37A1 IHC expression.

\begin{tabular}{|c|c|c|c|c|}
\hline \multirow[b]{2}{*}{ Variables } & \multicolumn{4}{|c|}{ SLC37A1 IHC } \\
\hline & Total (n) & Positive (n) & Negative (n) & P-value \\
\hline Total & 231 & 157 & 74 & \\
\hline Age, (years) & & & & 0.542 \\
\hline$\geq 60$ & 162 & 108 & 54 & \\
\hline$<60$ & 69 & 49 & 20 & \\
\hline Sex & & & & 1 \\
\hline Male & 136 & 92 & 44 & \\
\hline Female & 95 & 65 & 30 & \\
\hline Stage & & & & 0.479 \\
\hline 0 & 5 & 4 & 1 & \\
\hline I & 36 & 23 & 13 & \\
\hline II & 85 & 58 & 27 & \\
\hline III & 67 & 42 & 25 & \\
\hline IV & 38 & 30 & 8 & \\
\hline Tumor location & & & & 0.179 \\
\hline Right & 78 & 58 & 20 & \\
\hline Left & 133 & 99 & 54 & \\
\hline Histology & & & & 0.479 \\
\hline Well-differentiated & 100 & 64 & 36 & \\
\hline Moderately-differentiated & 102 & 74 & 31 & \\
\hline Poorly-differentiated & 7 & 4 & 3 & \\
\hline Mucinous & 19 & 15 & 4 & \\
\hline Depth & & & & 0.888 \\
\hline $\mathrm{T} 1$ & 28 & 18 & 10 & \\
\hline $\mathrm{T} 2$ & 27 & 18 & 9 & \\
\hline T3 & 159 & 108 & 51 & \\
\hline $\mathrm{T} 4$ & 17 & 13 & 4 & \\
\hline Lymphatic invasion & & & & 0.729 \\
\hline Absent & 47 & 31 & 16 & \\
\hline Present & 184 & 126 & 58 & \\
\hline Venous invasion & & & & 0.034 \\
\hline Absent & 46 & 25 & 21 & \\
\hline Present & 185 & 132 & 53 & \\
\hline Lymph node metastasis & & & & 0.315 \\
\hline Negative & 127 & 97 & 40 & \\
\hline Positive & 94 & 60 & 34 & \\
\hline Liver metastasis & & & & 0.013 \\
\hline Negative & 200 & 130 & 70 & \\
\hline Positive & 31 & 27 & 4 & \\
\hline
\end{tabular}

IHC, immunohistochemistry; SLC37A1, solute carrier family 37 member 1.

in multivariate models (Table IV), demonstrating the potential for SLC37A1 as a useful prognostic biomarker for CRC.

Knockdown of SLC37A1 decreases sialyl Lewis A and Xexpression in colon cancer cells. To evaluate the role of SLC37A1 in glycolipid metabolism, gene knockdown technology was used to investigate sialyl Lewis A and X expression. SLC37A1 expression in the 12 colon cancer cell lines (SW620, LoVo, SW480, HCT116, SW48, HCT15, RKO, SW837, COLO-201, COLO-205, LS174T and LS180) used in the present study was evaluated in order to select appropriate cells for the experiment (Fig. 2A). SLC37A1 was then knocked down by siRNA oligonucleotide in LS180 cells (SLC37A1-siRNA), which originally expressed upregulated SLC37A1 (Fig. 2B). 
Table IV. Univariate and multivariate Cox regression analyses of SLC37A1 expression levels and other clinical covariates.

\begin{tabular}{|c|c|c|c|c|}
\hline \multirow[b]{2}{*}{ Clinical covariates } & \multicolumn{2}{|c|}{ Univariate analysis } & \multicolumn{2}{|c|}{ Multivariate analysis ${ }^{\mathrm{a}}$} \\
\hline & $\operatorname{HR}(95 \% \mathrm{CI})$ & P-value & $\operatorname{HR}(95 \% \mathrm{CI})$ & P-value \\
\hline SLC37A1 expression (positive vs. negative) & $2.25(1.04-5.59)$ & 0.04 & $2.02(0.91-5.14)$ & 0.09 \\
\hline Age $(\geq 65$ years vs. $65<$ years $)$ & $1.18(0.60-2.36)$ & 0.63 & & \\
\hline Sex (male vs. female) & $0.89(0.46-1.77)$ & 0.74 & & \\
\hline Location (right vs. left) & $0.97(0.44-1.98)$ & 0.94 & & \\
\hline Stage (II-IV vs. 0-I) & $8.83(1.91-157)$ & $0.18 \times 10^{-2}$ & $0.66 \times 10^{10}(1.07-37.0)$ & 0.04 \\
\hline Histology (others vs. well) ${ }^{\mathrm{b}}$ & $3.53(1.63-8.79)$ & $0.09 \times 10^{-2}$ & $2.90(1.33-7.26)$ & 0.01 \\
\hline ly (present vs. absent) & $3.25(1.16-13.6)$ & 0.02 & $1.11(0.38-4.72)$ & 0.87 \\
\hline v (present vs. absent) & $5.16(1.56-31.8)$ & $0.04 \times 10^{-1}$ & $2.21(0.64-14.0)$ & 0.24 \\
\hline n (positive vs. negative) & $4.47(2.24-9.51)$ & $<0.01 \times 10^{-2}$ & $3.79(1.81-8.53)$ & $0.03 \times 10^{-2}$ \\
\hline
\end{tabular}

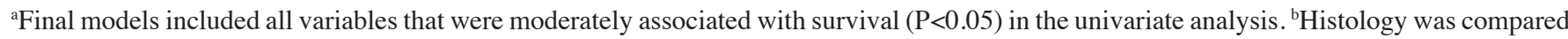
between others (moderately- and poorly-differentiated and mucinous) vs. well (well-differentiated). HR, hazard ratio; CI, confidence interval; SLC37A1, solute carrier family 37 member 1; ly, lymphatic invasion; v, venous invasion; n, node stage.

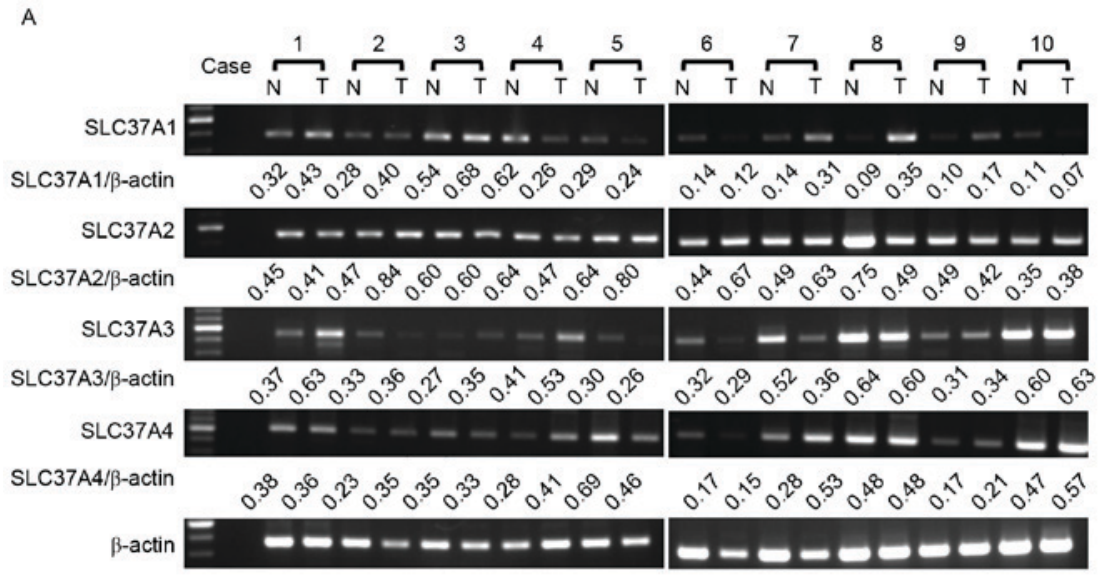

B

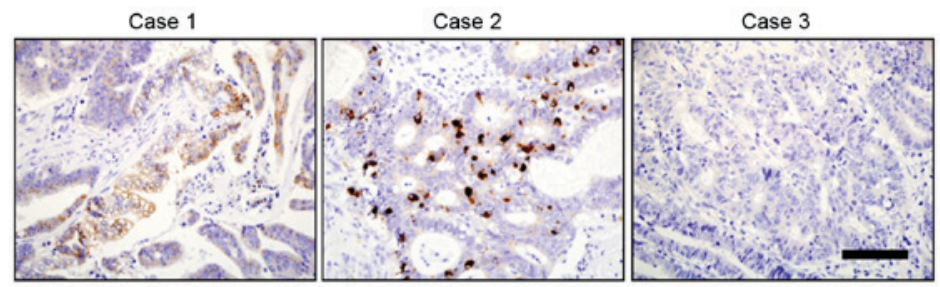

C

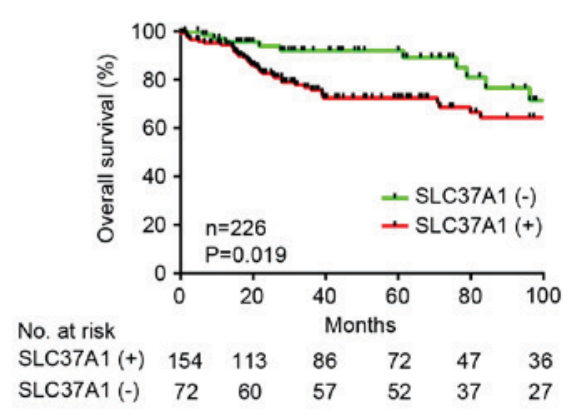

Figure 1. Expression of SLC37 family genes in patients with CRC. (A) Messenger RNA expression of SLC37 genes in patients with CRC. In total, 10 representative cases of tumor and non-tumor cells were analyzed by reverse transcription-quantitative polymerase chain reaction. $\beta$-actin was used as a loading control. Gene expression was quantified by the National Institutes of Health software ImageJ version 1.51k. (B) Immunohistochemical staining of SLC37A1 in CRC. Representative image of positive (cases 1 and 2) and negative (case 3) staining are shown. Scale bar, $100 \mu \mathrm{m}$. (C) Kaplan-Meier survival of 226 available CRC cases of the present cohort stratified by SLC37A1 tumor expression. CRC, colorectal cancer. T, tumor; N, non-tumor; SLC37A1, solute carrier family 37 member 1 . 
A

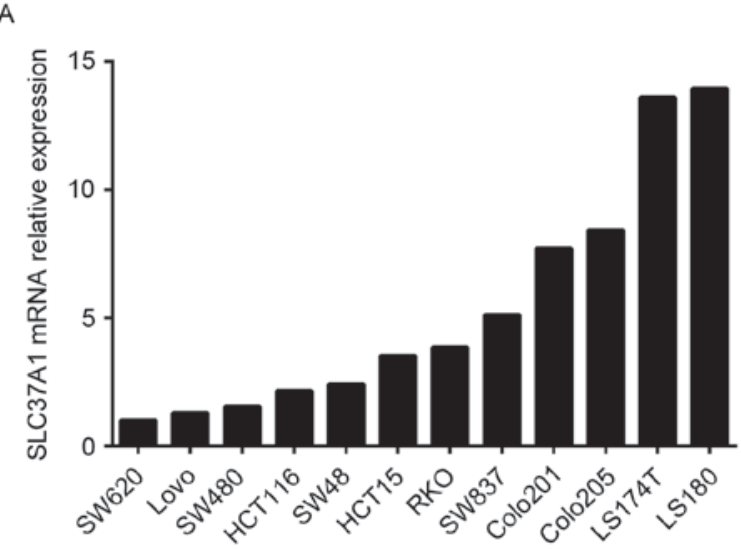

B

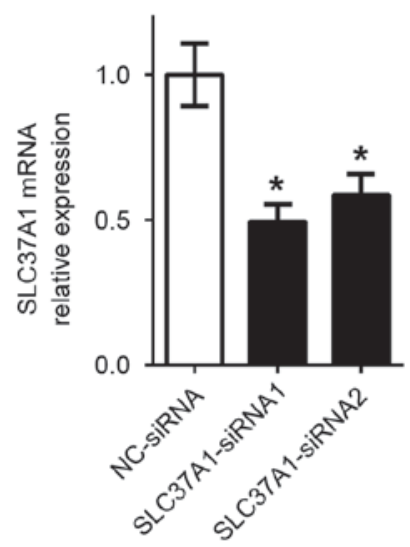

C
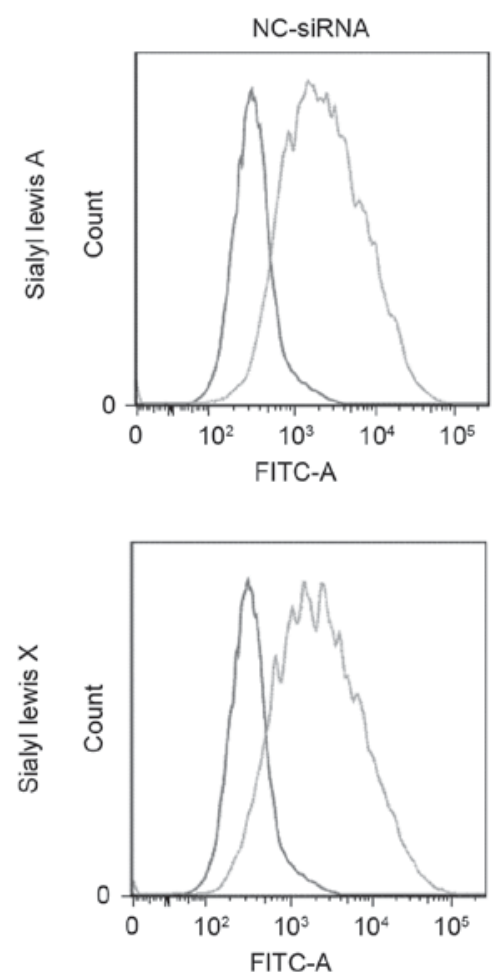
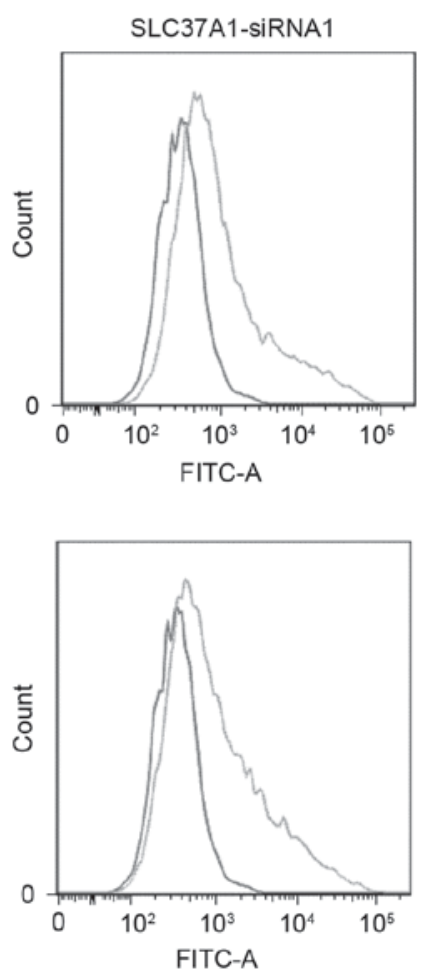

Figure 2. In vitro effects of SLC37A1 knockdown. (A) Expression of SLC37A1 in colon cancer cell lines. Relative SLC37A1 mRNA expression levels to SW620 (expression in SW620 cells was considered as 1) are shown (normalized to $\beta$-actin). (B) Expression of SLC37A1 in SLC37A1-knockdown LS180 cells (SLC37A1-siRNA1 and SLC37A1-siRNA2) and negative control cells (NC-siRNA). Data are presented as the mean \pm standard deviation. "P<0.05. (C) Flow-cytometric analysis of sialyl Lewis A and sialyl Lewis X expression in negative control cells (NC-siRNA; blue line in upper panels) and SLC37A1-knockdown LS180 cells (SLC37A1-siRNA1; blue line in lower panels). The red line represents the staining control (both upper and lower panels). FITC, fluorescein isothiocyanate; siRNA, small interfering RNA; NC, negative control; SLC37A1, solute carrier family 37 member 1.

While no morphological changes were observed in the SLC37A1-knockdown cells, the expression of sialyl Lewis A and sialyl Lewis $\mathrm{X}$ was markedly reduced compared with that in NC-siRNA cells (Fig. 2C).

\section{Discussion}

In the present study, increased SLC37A1 expression was observed in patients with $\mathrm{CRC}$, which was associated with positive venous invasion and liver metastasis. The present study confirmed that the tumor expression of SLC37A1 is upregulated at the mRNA and protein levels in patients with CRC, whereas other SLC37A family genes are not upregulated. Furthermore, positive expression of SLC37A1 is associated with poor patient survival, indicating an oncogenic role of SLC37A1 in CRC.

SLC37A1, located at chromosome 21q22.3, is one of the SLC37 family genes, and is abundantly expressed in the adult kidney, bone marrow, intestine, spleen and liver (27). While the functional role of SLC37A1 has not yet been characterized, an association with glycolipid metabolism has been suggested (28). SLC37A1 shares $30 \%$ of sequence similarity 
with the glycerol-3-phophate (G3P) transporter; therefore, SLC37A1 may be a G3P transporter $(19,27)$. By contrast, SLC37A1 also appears to function as a G6P transporter (29), indicating that even its fundamental role as a transporter remains controversial. It has been reported that SLC37A1 appears to be involved in phospholipid biosynthesis, indicating that SLC37A1 may accelerate cell proliferation (30). It appears that cells express SLC37A1 transporters to gain drug resistance, but the cell viability did not significantly differ between LS180 cells with high SLC37A1 expression and SW480 cells with low SLC37A1 expression (data not shown). In vitro experiments revealed that the expression of SLC37A1 was upregulated by epidermal growth factor (EGF) via the EGF receptor (EGFR)/mitogen-activated protein kinase/Fos transduction pathway in breast cancer cell lines (30). Since SLC37A1 expression was not associated with EGFR expression by IHC evaluation in the present cohort (data not shown), SLC37A1 may be upregulated by the downstream factor of EGFR signaling. The EGFR signaling pathway is also activated by KRAS mutations in CRC, indicating that SLC37A1 upregulation is involved in CRC tumor development. Approximately $50 \%$ of CRC cases have KRAS mutations (31), and they do not respond to anti-EGFR antibody therapy (32). This means that upstream inhibition of EGFR has no therapeutic effect in CRC cases with KRAS mutations (32). In addition, thus far, KRAS mutation has not served as a direct druggable target $(33,34)$. Therefore, to identify a candidate therapeutic target downstream of KRAS is reasonable.

Sialyl Lewis A and sialyl Lewis $\mathrm{X}$ are ligands for the adhesion molecule E-selectin, which is expressed in vascular endothelial cells and is associated with tumor metastasis, recurrence and overall survival in various patients with cancer (35). Our previous study reported that sialyl Lewis $\mathrm{A}$ and sialyl Lewis $\mathrm{X}$ were significantly induced by upregulation of uridine diphosphate-galactose transporter and hypoxia $(26,36)$. These carbohydrate antigens are responsible for the adhesion of cancer cells to the endothelium during metastasis (37). In the present study, positive SLC37A1 expression in CRC tumor tissue was associated with the presence of liver metastasis, indicating that SLC37A1 performs an important role in the hematogenous metastasis of CRC. Therefore, SLC37A1 was knocked down in colon cancer cells, and a significant reduction of sialyl Lewis A and sialyl Lewis $\mathrm{X}$ expression was observed. These results indicated that induced SLC37A1 expression in CRC tumor tissue accelerates cancer metastasis and may be a therapeutic target in patients with CRC.

In conclusion, to the best of our knowledge, the present study is the first to report that SLC37A1 is upregulated in CRC and is associated with poor patient outcomes. Additional studies are required to investigate the possibility of SLC37A as a candidate therapeutic target or biomarker to detect high-risk patients.

\section{Acknowledgements}

The present study was supported by the Japan Society for the Promotion of Science via the Grants-in-Aid for Scientific Research KAKENHI program (grant no. 15k10143).

\section{References}

1. Siegel RL, Miller KD and Jemal A: Cancer statistics, 2016. CA Cancer J Clin 66: 7-30, 2016.

2. Chen W, Zheng R, Zhang S, Zhao P, Li G, Wu L and He J: Report of incidence and mortality in China cancer registries, 2009. Chin J Cancer Res 25: 10-21, 2013.

3. Katanoda K, Hori M, Matsuda T, Shibata A, Nishino Y, Hattori M, Soda M, Ioka A, Sobue T and Nishimoto H: An updated report on the trends in cancer incidence and mortality in Japan, 1958-2013. Jpn J Clin Oncol 45: 390-401, 2015.

4. Venook AP, Weiser MR and Tepper JE: Colorectal cancer: All hands on deck. Am Soc Clin Oncol Educ Book 83-89, 2014.

5. Carrato A: Adjuvant treatment of colorectal cancer. Gastrointest Cancer Res 2 (4 Suppl): S42-S46, 2008.

6. Longley DB, Harkin DP and Johnston PG: 5-fluorouracil: Mechanisms of action and clinical strategies. Nat Rev Cancer 3: 330-338, 2003.

7. de Gramont A, Figer A, Seymour M, Homerin M, Hmissi A, Cassidy J, Boni C, Cortes-Funes H, Cervantes A, Freyer G, et al: Leucovorin and fluorouracil with or without oxaliplatin as first-line treatment in advanced colorectal cancer. J Clin Oncol 18: 2938-2947, 2000.

8. Douillard JY, Cunningham D, Roth AD, Navarro M, James RD, Karasek P, Jandik P, Iveson T, Carmichael J, Alakl M, et al: Irinotecan combined with fluorouracil compared with fluorouracil alone as first-line treatment for metastatic colorectal cancer: A multicentre randomised trial. Lancet 355: 1041-1047, 2000.

9. Goldberg RM, Sargent DJ, Morton RF, Fuchs CS, Ramanathan RK, Williamson SK, Findlay BP, Pitot HC and Alberts SR: A randomized controlled trial of fluorouracil plus leucovorin, irinotecan, and oxaliplatin combinations in patients with previously untreated metastatic colorectal cancer. J Clin Oncol 22: 23-30, 2004.

10. Tournigand C, André T, Achille E, Lledo G, Flesh M, Mery-Mignard D, Quinaux E, Couteau C, Buyse M, Ganem G, et al: FOLFIRI followed by FOLFOX6 or the reverse sequence in advanced colorectal cancer: A randomized GERCOR study. J Clin Oncol 22: 229-237, 2004.

11. $\mathrm{Hu} \mathrm{T}, \mathrm{Li} \mathrm{Z}, \mathrm{Gao} \mathrm{CY}$ and $\mathrm{Cho} \mathrm{CH}$ : Mechanisms of drug resistance in colon cancer and its therapeutic strategies. World J Gastroenterol 22: 6876-6889, 2016.

12. Lin L, Yee SW, Kim RB and Giacomini KM: SLC transporters as therapeutic targets: Emerging opportunities. Nat Rev Drug Discov 14: 543-560, 2015.

13. Dean M, Rzhetsky A and Allikmets R: The human ATP-binding cassette $(\mathrm{ABC})$ transporter superfamily. Genome Res 11: 1156-1166, 2001

14. Szakács G, Annereau JP, Lababidi S, Shankavaram U, Arciello A, Bussey KJ, Reinhold W, Guo Y, Kruh GD, Reimers M, et al: Predicting drug sensitivity and resistance: Profiling ABC transporter genes in cancer cells. Cancer Cell 6: 129-137, 2004.

15. Higgins CF: Multiple molecular mechanisms for multidrug resistance transporters. Nature 446: 749-757, 2007.

16. Shukla S, Chen ZS and Ambudkar SV: Tyrosine kinase inhibitors as modulators of $\mathrm{ABC}$ transporter-mediated drug resistance. Drug Resist Updat 15: 70-80, 2012.

17. Gottesman MM, Fojo T and Bates SE: Multidrug resistance in cancer: Role of ATP-dependent transporters. Nat Rev Cancer 2: 48-58, 2002.

18. Lopez-Lopez E, Ballesteros J, Piñan MA, Sanchez de Toledo J, Garcia de Andoin N, Garcia-Miguel P, Navajas A and Garcia-Orad A: Polymorphisms in the methotrexate transport pathway: A new tool for MTX plasma level prediction in pediatric acute lymphoblastic leukemia. Pharmacogenet Genomics 23: 53-61, 2013.

19. Chou JY and Mansfield BC: The SLC37 family of sugar-phosphate/phosphate exchangers. Curr Top Membr 73: 357-382, 2014.

20. Levine AJ and Puzio-Kuter AM: The control of the metabolic switch in cancers by oncogenes and tumor suppressor genes. Science 330: 1340-1344, 2010.

21. Chen JQ and Russo J: Dysregulation of glucose transport, glycolysis, TCA cycle and glutaminolysis by oncogenes and tumor suppressors in cancer cells. Biochim Biophys Acta 1826: 370-384, 2012.

22. Sobin LH and Compton CC: TNM seventh edition: What's new, what's changed: Communication from the international union against cancer and the American joint Committee on cancer. Cancer 116: 5336-5339, 2010. 
23. Sobin LH, Gospodarowicz M and Wittekind Ch (eds): International Union Against Cancer TNM Classification of Malignant Tumors. 7th edition. Wiley-Blackwell, Oxford, UK, 2009.

24. Okano M, Kumamoto K, Saito M, Onozawa H, Saito K, Abe N, Ohtake T and Takenoshita S: Upregulated Annexin A1 promotes cellular invasion in triple-negative breast cancer. Oncol Rep 33: 1064-1070, 2015.

25. Livak KJ and Schmittgen TD: Analysis of relative gene expression data using real-time quantitative PCR and the 2(-Delta Delta C(T)) Method. Methods 25: 402-408, 2001

26. Koike T, Kimura N, Miyazaki K, Yabuta T, Kumamoto K, Takenoshita S, Chen J, Kobayashi M, Hosokawa M, Taniguchi A, et al: Hypoxia induces adhesion molecules on cancer cells: A missing link between Warburg effect and induction of selectin-ligand carbohydrates. Proc Natl Acad Sci USA 101: 8132-8137, 2004.

27. Bartoloni L, Wattenhofer M, Kudoh J, Berry A, Shibuya K, Kawasaki K, Wang J, Asakawa S, Talior I, Bonne-Tamir B, et al: Cloning and characterization of a putative human glycerol 3 -phosphate permease gene (SLC37A1 or G3PP) on 21q22.3. Mutation analysis in two candidate phenotypes, DFNB10 and a glycerol kinase deficiency. Genomics 70: 190-200, 2000.

28. Bartoloni L and Antonarakis SE: The human sugar-phosphate/phosphate exchanger family SLC37. Pflugers Arch 447: 780-783, 2004.

29. Pan CJ, Chen SY, Jun HS, Lin SR, Mansfield BC and Chou JY: SLC37A1 and SLC37A2 are phosphate-linked, glucose-6-phosphate antiporters. PLoS One 6: e23157, 2011.

30. Iacopetta D, Lappano R, Cappello AR, Madeo M, De Francesco EM, Santoro A, Curcio R, Capobianco L, Pezzi V, Maggiolini $M$ and Dolce V: SLC37A1 gene expression is up-regulated by epidermal growth factor in breast cancer cells. Breast Cancer Res Treat 122: 755-764, 2010.
31. Cancer Genome Atlas N: Comprehensive molecular characterization of human colon and rectal cancer. Nature 487: 330-337, 2012.

32. Siddiqui AD and Piperdi B: KRAS mutation in colon cancer: A marker of resistance to EGFR-I therapy. Ann Surg Oncol 17: 1168-1176, 2010.

33. Dietlein F, Kalb B, Jokic M, Noll EM, Strong A, Tharun L, Ozretic L, Künstlinger H, Kambartel K, Randerath WJ, et al: A Synergistic Interaction between Chk1- and MK2 Inhibitors in KRAS-Mutant Cancer. Cell 162: 146-159, 2015.

34. Zhu Z, Aref AR, Cohoon TJ, Barbie TU, Imamura Y, Yang S, Moody SE, Shen RR, Schinzel AC, Thai TC, et al: Inhibition of KRAS-driven tumorigenicity by interruption of an autocrine cytokine circuit. Cancer Discov 4: 452-465, 2014.

35. Liang JX, Liang Y and Gao W: Clinicopathological and prognostic significance of sialyl Lewis $\mathrm{X}$ overexpression in patients with cancer: A meta-analysis. Onco Targets Ther 9: 3113-3125, 2016.

36. Kumamoto K, Goto Y, Sekikawa K, Takenoshita S, Ishida N, Kawakita M and Kannagi R: Increased expression of UDP-galactose transporter messenger RNA in human colon cancer tissues and its implication in synthesis of Thomsen-Friedenreich antigen and sialyl Lewis A/X determinants. Cancer Res 61: 4620-4627, 2001.

37. Takada A, Ohmori K, Yoneda T, Tsuyuoka K, Hasegawa A, Kiso M and Kannagi R: Contribution of carbohydrate antigens sialyl Lewis A and sialyl Lewis X to adhesion of human cancer cells to vascular endothelium. Cancer Res 53: 354-361, 1993.

This work is licensed under a Creative Commons Attribution-NonCommercial-NoDerivatives 4.0 International (CC BY-NC-ND 4.0) License. 